\title{
0942 TRADITIONS IN IMPLEMENTATION: CASE STUDY OF FALLS PREVENTION IN A RURAL HEALTH AUTHORITY
}

J Brown*, S Baker, I Zwerling Correspondence: Dalhousie University, School of Occupational Therapy, 5869 University Avenue Forrest Building, Room 215, Dalhousie University, Halifax, Nova Scotia B3H 3J5, Canada

10.1136/ip.2010.029215.942

Introduction Rural Health Authorities are challenged to address the complex issue of falls in aging populations. While extensive evidence exists regarding successful falls prevention interventions, there is little information about planning and implementation at an organisational level. This presentation outlines results from research examining falls prevention at a rural health authority.

Method Case study methodology was applied in a phased approach examining development and implementation of the falls prevention strategy. Data collection focused barriers and facilitators experienced by team members, and suggestions to improve processes used. Data collection methods included review of key documents and field notes, participant observation and interviews. The interviews were reviewed, coded and analysed to extract themes.

Results The process of strategy development is outlined. The themes for planning reveal the lack of consensus regarding what constitutes a planning process, non-linearity of planning and implementation, and tremendous complexity of barriers and supports. Analysis of implementation identifies themes of the importance of culture within teams and within organisations, a dynamic tension between policy makers and healthcare makers, and competing demands for attention at both individual and organisational levels. Facilitation strategies that contribute to changing everyday practice are discussed, as well as sources of resistance to change.

Conclusions This research provides insight for health organisations addressing falls in aging populations, particularly in rural areas which lack well developed resources and traditions for implementation of evidence in routine practices. Translating evidence into everyday practice for injury prevention is complex, and easily overwhelmed by competing demands. 